\title{
Applying PLU Rules on Different BIM/GIS Interoperability Approaches
}

\author{
Elio HBEICH ${ }^{1,2}$, Ana ROXIN ${ }^{2}$, Nicolas BUS ${ }^{1}$ \\ ${ }^{1}$ Information System and Applications Division, CSTB, Sophia Antipolis 06560, France \\ ${ }^{2}$ University of Bourgogne Franche-Comté (UBFC) - LIB EA7534 \\ Elio.hbeich@cstb.fr, ana-maria.roxin@ubfc.fr, nicolas.bus@cstb.fr
}

Compliance checking for building models, cities and territories involves formalizing a set of model schemas and constraints. In this context, we aim to achieve two goals: (1) achieve semantic interoperability between BIM (IFC) and GIS (CityGML), which help us represent building information in its details and its surrounding environment; (2) adapting of PLU rule into semantic queries. In this article, we will introduce (A) BIM and GIS interoperability approaches, and (B) connecting the interoperable models, with PLU levels. Our approach addresses (Prob1) interoperability between IFC and CityGML on building scale model, and (Prob2) the correlation between PLU rules and the multiscale model.

Keywords: BIM, GIS, CityGML, IFC, Interoperability, PLU

\section{$\mathbf{1}^{1}$} Introduction

Today we are witnessing a fourth industrial revolution (Industry 4.0) marked by the concept of factories in which machines are augmented with wireless connectivity and sensors, connected to a system that can visualize the entire production line, control, and decide on its own. In addition, it allows communication between humans as well as machines [1]. Due to the increasing number of elements and systems, complex and heterogeneous enterprise systems need to be considered, the use of heterogeneous systems generates an environment that integrates the systems forming a System-of-Systems (SoS). This revolution affects the urban scopes, and it is manifesting by the collaboration between multiple stakeholders and territorial communities, where innovative rules and norms aim to integrate different systems that conceive multilevel, multi-scale solutions addressing common challenges. In this paper, we are going to discuss the making of a system that integrates the following three environments/systems: Building Information Model (BIM), Geographic Information Model (GIS) and Local Urban Plan (PLU). In this context, we aim to achieve two goals: (1) achieve semantic interoperability between BIM (IFC) and GIS (CityGML), which help us represent building information in its details and its surrounding environment; (2) adapting PLU rule into se- mantic queries. In this article, we will introduce (A) BIM and GIS interoperability approaches, and (B) connecting the interoperable models, with PLU. Our approach addresses (Prob1) interoperability between IFC and CityGML on building scale model, and (Prob2) the correlation between PLU rules and the multiscale model. The article is divided as follows: section 2 introduces Local Urban Plan, BIM and GIS information models, interoperability approaches, section 3 presents previous work related to transforming urban regulations rules into semantic queries and the integrating approaches between IFC, CityGML, section 4 introduces research issues we are aiming to address while section 5 describes three conceptual interoperability approaches, section 6 presents semantic checking in each interoperability approach and finally conclusion and future work are discussed in section 7 .

\section{Background}

\subsection{Local Urban Plan (PLU)}

In France, urban construction, renovation or landscaping projects always require checking the considered municipality PLU (Local Urban Plan). This document indicates the urban planning rules governing the considered municipality, whether in terms of urban development, preservation of spaces and architectural prescriptions. The objective of the PLU is to improve a) the living spaces of inhabitants 
concerning a sustainable idea of urbanism; b) travel and transportation by taking into account infrastructure projects, c) neighbourhoods restructuring while preserving natural heritage, etc. It guarantees the balance between urban development and the preservation of natural spaces in a perspective of sustainable development [2]. A PLU defines 4 main types of areas (see Fig. 1):

- Urban areas (U): Such zone corresponds to areas of the city that are urbanized or in the process of urbanization. Which means that the public networks (water, electricity, etc.) and the public road connects each parcel. That is why it is the most favourable area to build since it already has the necessary networks for viable land. In addition, when looking at the urban area of the PLU of medium or large cities, you will notice that zone $\mathrm{U}$ is itself divided into sectors: UA, UB, UC, UD, UE, UL, UI, UP, UT, UV, and UY [3], where each sector/sub-zone have certain characteristics

- Areas to be urbanized (AU): known as "AU zones", these areas are defined by
Article R. 151-2029 of the French urban planning code. According to the French legislation, such area is considered as a zone intended to be open to public urbanization [4].

- Agricultural zones (A): These are "areas of the municipality, equipped or not, to protect because of the potential agronomic, biological or economic agricultural land" (article R. 151-2230 of the French urban planning code) [5]. It is a strict and supervised regime, only the constructions or installations necessary for the public services and the agricultural exploitation are authorized there.

- Natural and forest areas (N): These are the "sectors of the commune, equipped or not, to be protected because of the quality of the sites, the natural environments, the landscapes and their interest, in particular from the aesthetic, historical or ecological point of view, or from the existence of forest exploitation, or of their character as natural spaces" (article R. 151-2431 of the urban planning code) [6].

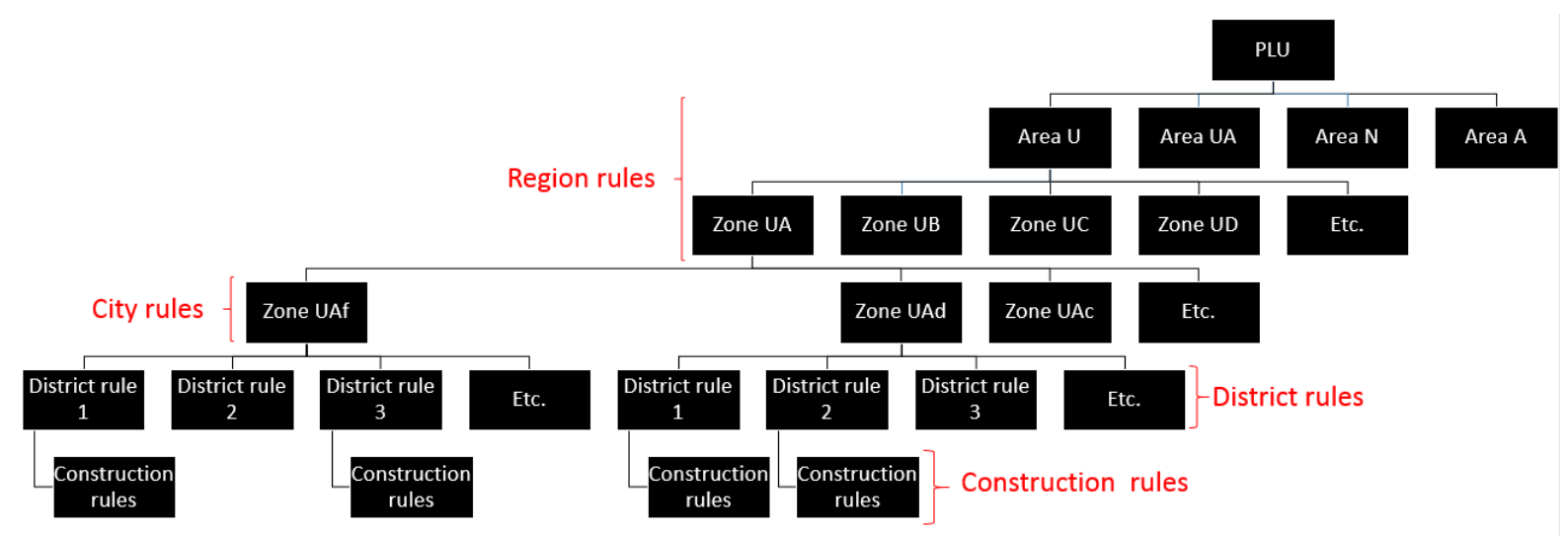

Fig. 1. PLU Hierarchy

\subsection{Building Information Modelling (BIM)}

"Building" goes beyond what one would consider a building, including infrastructure elements such as roads, tunnels, bridges, etc. It aims at supporting sharing reliable information throughout the lifecycle of the considered built element, from design to demolition. Such digital model is a representation of the physical and functional characteristics of the built element. Also, BIM data exchange standard is represented by IFC. It is an object-oriented open standard initiated by buildingSMART in 1994. It has now become a formally registered international standard as ISO 16739:2013 [7]. Thus, IFC (ISO 16739-1) data is structured according to the EXPRESS specification [8], where it is divided into four layers (see Figure 2): 
- Domain Layer: contains entity definitions for concepts specific to individual domains such as architecture, structural engineering facilities and management

- Interoperability layer: comprises entity categories that are commonly used and shared between multiple building construction and facilities management application.
- Core layer: process and control related concepts such as task, procedure, work schedule, performance history, work approval, and defines abstract building components such as space site building, building element, annotation.

- Resource layer: contains basic properties such as geometry, material, quality, measurement, date and time, cost, actors, roles.

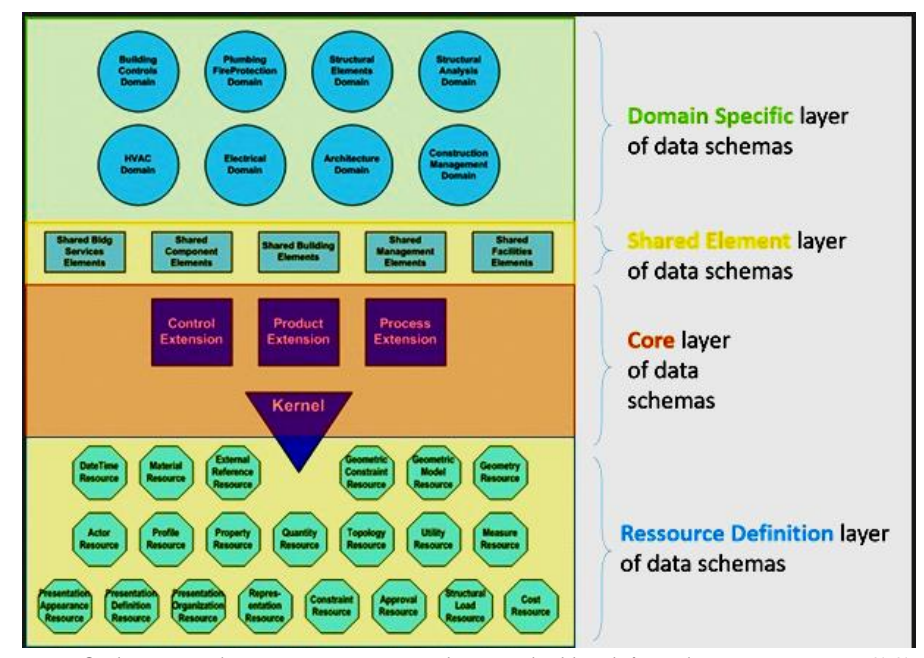

Fig. 2. IFC layers of data schemas [7] and modelled in the EXPRESS Schema [8].

\subsection{Geographic Information System (GIS)}

GIS is a computer-based tool that allows you to create, manipulate, analyse, store and display information based on its location. GIS makes it possible to integrate different types of geographic information, such as digital maps, aerial photographs, satellite images and global positioning system data (GPS), along with associated tabular database information. One of the most GIS used tools is CityGML (City Geography Markup Language) [9]. CityGML is implemented as an application schema that defines an outline, diagram, or model to describe the structure of different types of data in GML3. CityGML is based on the ISO 191xx family, with inputs from other standardization bodies such as OGC (Open Geospatial Consortium), W3C (World Wide Web Consortium), and OASIS (Organization for the Advancement of Structured Information Standards). In addition, CityGML uses taxonomies and aggregations such as: digital terrain models (DTM), regular raster, break and skeleton lines, mass points, sites, vegetation, water bodies, etc. Furthermore, CityGML comprises a multi-scale abstraction model defining the so-called Levels of Detail (LODs) [9], as described below:

- LOD0 is a two and a half dimensional

- LOD1 adds to LOD0 block models comprising buildings with flat roof structures

- LOD2 is LOD1 with different roof structures and boundary surfaces

- LOD3 adds external details, e.g. walls and roofs along with doors and windows

- LOD4 contains internal details of a building for example chairs, etc.

Finally, Application Domain Extensions (ADE) are provided as specific "hooks" in the CityGML schema that allows defining application-specific extensions of the CityGML model. For example, in the context of noise pollution simulations, building reflection, emission points, habitants, etc. are added as properties to the _AbstractBuilding class. 


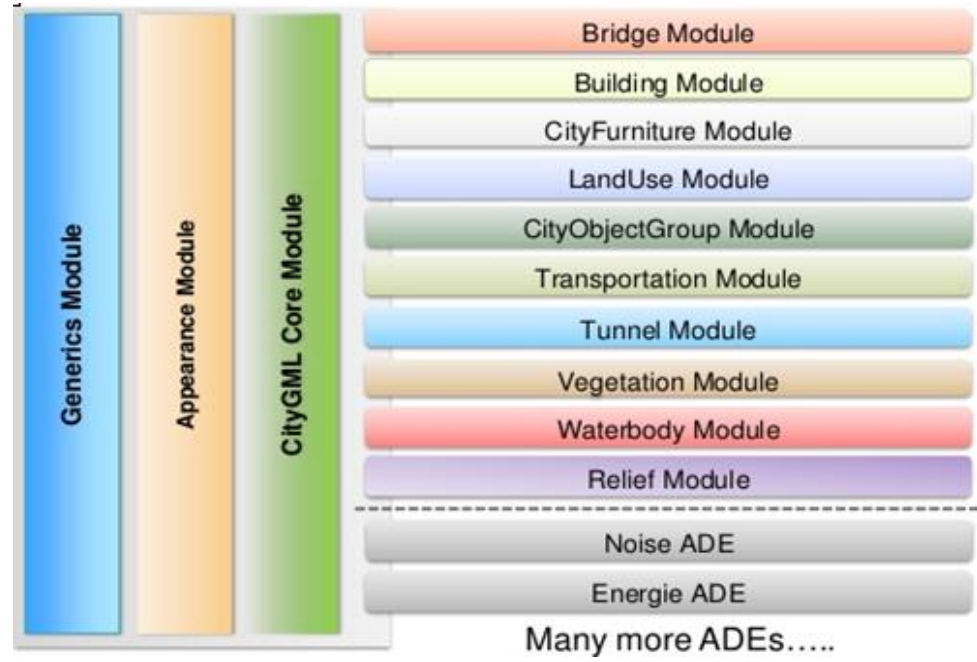

Fig. 3. CityGML Modular standard

\subsection{Interoperability Concept}

Interoperability is defined as the "capability to communicate, execute programs, or transfer data among various functional units in a manner that requires the user to have little or no knowledge of the unique characteristics of those units" [10]. This definition implies that interoperable systems can either exchange information or be accessed with a single method. Hence achieving interoperability allows parts of the system and the overall system to cooperate seamlessly to reach an appropriate result. Interoperability framework identifies three main levels of interoperability, namely: data, syntactic, and semantic interoperability. These layers are connected and build upon each other, lower level providing elements required by upper levels functionalities [11]:

- Data/physical interoperability: usually associated with hardware/software components, systems, and platforms that cover the technical issues of linking computer systems and services. It mainly concerns protocol and infrastructure. It has been long resolved with the adoption of hardware standards such as Ethernet [12]; along with standard protocols for lower layers of the ISO network architecture e.g. TCP/IP [13] [14], etc.

- Syntactic interoperability: addresses the syntax of messages exchanged among
CSPs considered artefacts. The related issues have been resolved through the adoption of XML and related syntax standards e.g. HTML, WSDL [15] and SOAP [16]

- Semantic interoperability: addresses the meaning of the messages exchanged and related issues have not yet been resolved by existing standards and approaches. Semantic Web standards and languages allow specifying such meaning, through formal and explicit specifications of conceptualisations e.g. ontologies

Ontologies alone don't resolve the issue of interoperability as they are autonomous from each other, which is why semantic connections must be made to distinguish and adjust concepts and relations between those ontologies. ISO 11354 [17] defined 3 types of Semantic interoperability:

- Integrated approach: require that every component must follow a common form. Which define a degree of expressiveness permitting to catch the particular subtleties of the components, particularly those affecting interoperability.

- Unified approaches: require a representative meta-model. It builds semantic equivalence among concepts or substances. In addition, all considered models must follow the defined meta-model. 
- Federated approaches: implies that no collaborator forces their models, languages, or strategies for work. They mostly apply to contexts where the entities considered for interoperability depend on two divers or complex vocabularies or methodologies. To arrive at interoperability in such a unique circumstance, mappings must be indicated among input and output information of the considered entities.

\subsection{Conclusion}

Based on what has been previously discussed we have concluded the following:

- We have a multi-area type, where each area is divided into multi-zones where different regulations are applied to check the compliance of city elements in the zone, e.g. buildings, transportation networks. Which indicates a certain granularity and aggregation for the regulations contained in the PLU. To achieve automated regulatory compliance checking for a construction project, we need to automatically extract requirements from various construction regulation documents and transform these rules into a formalized syntax that enables machine automated reasoning. To reach our goal, we seek to express our set of rules in a language that can be handled and interpreted by a machine. As all considered urban models are converted into ontologies, we aim to transform natural language rule into semantic queries such as SPARQL, as it is the equivalent of SQL for ontologies.

- We have noticed the need for a multiscale model that contains necessary information related to building, district, city and urban scale. Two information models are considered to generate the desirable multi-scale model: Building Information Modelling (BIM) and Geographic Information System (GIS). In this article, we are describing three approaches of BIM and GIS interoperability
3 Previous Work Related to BIM/GIS Interoperability and Transforming Textual Regulation into Formal Rules

\subsection{Related Work for BIM/GIS Integration}

In this section, we are going to introduce previous work addressing BIM/GIS integration/linkage:[21] introduces a semantic extension called Urban Information Modelling (UIM), that defines spatial, temporal and multi-representation concepts using extensible ontology. However, the main drawback of this approach persists in the usage of database to store the instances of the ontology. [22] solves the interoperability between BIM and GIS by applying the following steps: 1) transforming IFC to RDF, 2) transform GIS to $\mathrm{RDF}$, and finally 3) using the GMO (graph matching for ontologies) algorithm to map between the Ontologies. However, the algorithm needs more enhancement as it does not take into count all semantic information. Authors in [23] present a methodology to transform the IFC model into CityGML as a potential way to achieve interoperability between GIS and BIM. However, the methodology presented did not investigate a fully complex model, and many semantic information was lost in the process.

\subsection{Related Work for Adapting Regulations into Formal Rules}

In this section, we are going to introduce previous work addressing transforming textual regulation into formal rules:

In [29], the authors present an approach and a tool to assist in compiling regulations and technical documents. For this purpose, a domain ontology, OntoDT, was introduced to represent concepts involved in the description of technical documents and regulations. Combining with SBVR (Semantics of Business Vocabulary and Business Rules) and Semantic Web languages, a controlled vocabulary was defined, based on which regulatory constraints extracted from the Practical Guides, edited by CSTB (French Scientific and Technical Centre for Building), was adapted. The SBVR-based approach reduces ambiguity, RDF and SPARQL based formalization enable automating regulation conformance 
checking. The presented approach transforms textual rules into semi-formal rule than to formal rules.

In [30], the authors present an Information Transformation (ITr) methodology. It relies on a rule-based, semantic natural language processing (NLP) approach. A set of Semantic Mapping (SeM) rules and Conflict Resolution $(\mathrm{CoR})$ rules are used to enable the automation of the transformation process. Several syntactic text features (captured using NLP techniques) and semantic text features (captured using an ontology) are used in the SeM and CoR rules. A bottom-up method is leveraged to handle complex sentence components.

\subsection{Conclusion}

Based on the semantic interoperability approaches we are going to present the existing possibility to connect IFC and CityGML on building scale. Also, we are going to adapt the approach presented in [29] to transform textual rules into semantic queries and enhance it in future work.

\section{Research Issue Addressed In This Paper}

PLU regulations are aggregated on different scale: region, city, district and buildings. This points us toward the need of a multi-scale model that contains necessary information related to each scale. Based on that we have presented before we can conclude that IFC model represents the building scale and CityGML represents the region, city, and district scale. To be able to detect the non-compliant element in an urban, city, district and building scale we need to achieve interoperability between BIM and GIS on a semantic level (based on section 2.4) and transform the PLU rules into semantic queries such as SPARQL and finally apply the SPARQL queries on BIM and GIS to retrieve the non-compliant elements. In this section we are going to discuss interoperability issues between BIM and GIS, explain how regulations can be adapted into logical rules through logical languages e.g. SHACL/SWRL / RIF, and finally clarify why ontologies are suitable to solve our problems.

\subsection{BIM and GIS Interoperability Issues}

While BIM provides 3D perception and different functionalities to sort out and oversee immense volumes of information identified with structures, GIS situations are profoundly adaptable, well-prepared for multi-dimensional examination, and perfect for activities including multi-site environments. Even though, both models can benefit from one another, they were put in a state of competition. In addition, throughout their development no association or synchronization have been defined between them. Which created a gap between both models thus prevent us to check a building according to its urban environment. Bringing interoperability between BIM and GIS comes with several challenges such as:

- Coordinate systems and spatial referencing: GIS use two dimensional real world coordinates (RWC 9), it is based on a global spatial reference systems and use boundary representation, while BIM systems use three dimensional relative coordinates between objects, with a reference to RWC at root object and use boundary, Swept Solid and Constructive Solid Geometry representation.

- Temporal aspects: In BIM a building object is characterized by its geometrical representations and its non-geometrical properties. Such object can have several geometrical representations, where each correspond to a different point of view. However, no standard has defined any links between the geometrical representations, properties and the different levels of detail handled by GIS systems.

- Semantics: BIM and GIS use various vocabularies to illustrate their entities and properties. Yet there is no semantic layer defining standard vocabularies, relationships between BIM and GIS ontological models. Such semantic links are essential to achieve full semantic interoperability to map and synchronize the definition of entities, properties of each domain and map it to its equivalent. 
4.2 Adapting Regulation into Logical Rules Logic rules provide an adaptable language for communicating high-level of structured knowledge. It services to decompose a realworld situation and look for logical rules which may govern a situation. The main clue is to break the situation into smaller parts and then look for logical rules which we can model with a computer. In our situation the logical rule will contribute on dividing complex rules into smaller simpler rules or group multiple rules to answer a higher rule where each one verifies and checks a certain characteristics. Table. 1 represents an example, of logical rules using logical languages e.g. SHACL/SWRL / RIF.

Table 1. Example of regulating and logical rules

\begin{tabular}{|c|c|c|c|}
\hline PLU rules & Sub rules & SPARQL & SWRL \\
\hline $\begin{array}{l}\text { Rl: The re- } \\
\text { gion can } \\
\text { contain any } \\
\text { accommoda- } \\
\text { tion and } \\
\text { housing }\end{array}$ & none & geof:sfWithin(?IfcProject, ?_Site) & none \\
\hline $\begin{array}{l}\text { R2: Building } \\
\text { frontage } \\
\text { height } \\
\text { should not } \\
\text { be greater } \\
\text { than } 55 \mathrm{~m} \text { in } \\
\text { a UP sub } \\
\text { zone }\end{array}$ & $\begin{array}{l}\text { Divide into } 2 \text { rules: } \\
\text { Rule2.1: frontage } \\
\text { height }>55 \mathrm{~m} \\
\text { Rule2.2: building } \\
\text { contained in UP sub- } \\
\text { zone }\end{array}$ & $\begin{array}{l}\text { Rule2.1: ifcowl:IfcWall } \\
\text { ifcowl:height ?height } \\
\text { op:numeric-greater-than(?height, } \\
\text { 55m) } \\
\text { Rule2.2: geof:sfWithin } \\
\text { (ifcowl:IfcBuilding, } \\
\text { gml:Landuse) }\end{array}$ & $\begin{array}{l}\text { rule2.1 } \\
\text { rule2.2 } \\
\Rightarrow \mathrm{R} 2\end{array}$ \\
\hline
\end{tabular}

\subsection{Ontology}

"Ontology is an explicit and formal specification of a shared conceptualization. The term is borrowed from philosophy, where Ontology is a systematic account of Existence". Also, an ontology is a" formal, explicit specification of a shared conceptualization" [19]. Based on these definitions we are going to explain: what is a conceptualization? What is a proper formal, explicit specification? And why is 'shared' of importance?

\subsubsection{Conceptualization}

Conceptualization is an abstract, simplified view of some selected part of the world, containing the objects, concepts, and other entities that we wish to represent for some purpose. Where every knowledge base/system/level is committed to some conceptualization, explicitly or implicitly."

\subsubsection{Proper Formal, Explicit Specification} An ontology is identified as explicit when all concepts, properties and relations are stated clearly and in detail, leaving no room for confusion or doubt. Hence the ontology will gain clarity, coherence, and extensibility. An ontology is described as formal when it is easily understandable not only by human but also by machine, where the machine can handle such model specification.

\subsubsection{Why Shared is Important}

Because ontology captures the conceptual structure of the domain. Shared ontologies help in increasing the reuse of knowledge where, we can be able to define new terms for special uses based on the existing vocabulary, in a way that does not require the revision of the existing definitions. This characteristic enables us to use ontology in: information-retrieval systems, digital libraries, integration of heterogeneous information sources, etc. 
4.3.3 Why are Ontologies Pertaining for the Considered Context?

From a data perspective, the main objective is how to break up data silos and how to connect data sources spread across agencies, departments, and third-party providers to create actionable knowledge. Semantic web technologies solve these two challenges with a standards-based approach that has been widely implemented throughout the World Wide Web and in several enterprise use cases. A semantic layer on top of your content provides endless possibilities to develop urban applications within robust information architecture. Integration harmonizes data and metadata as a Knowledge Graph, which makes content from disparate systems easily accessible. This facilitates novel approaches to quality assurance and trust of information. Semantic information architecture provides along with quality, context and meaning to your data based on controlled vocabularies. Who it works? An ontology comprises a terminological model (TBox) which contains the formal definitions of the concepts relevant for the considered domain of discourse. The instantiation of these concepts results in an assertion model (ABox). The combination of terminologies and assertion boxes results in a so-called knowledge base (also called an ontology). Also, formal and explicit semantics allow implementing reasoning capabilities thus inferring new information from existing data. Several languages have been specified for defining ontologies. They are all based on the RDF graph model (RDF/RDF Schema [24]) and allow different levels of expressivity depending on description logic-based knowledge representation (the families and different profiles of Web Ontology Language [24]). Therefore, ontologies have been recognized by their ability to represent information and knowledge of various domains. For many authors, the use of ontologies improves interoperability among different information systems in general [25] [26] [27]. In addition, transmitting information between models requires the ability to create alignments between concepts/properties from one system to concepts/properties in the other one. Thus, the usage of ontologies will facilitate the matching mechanism. As a first step achieving interoperability between CityGML and IFC we are going to first to transform both models into Ontologies.

\section{Possible Approaches for implementing interoperability at an urban scale 5.1 Federation Approach}

In this section we are going to introduce the Federation Architecture for OWL Ontologies (FOWLA) and the conceptual approach to reach BIM and GIS by applying the same methodology of FOWLA.

\subsubsection{Federated Architecture for OWL On- tologies (FOWLA)}

Federation approach does not aim to integrate or unify CityGML and IFC models, it only seeks to generate strong and light coupling/connection between the two models. To do so we consider relying on an existing approach: federated architecture for OWL ontologies or FOWLA [28]. FOWLA is an approach relying on SWRL rules for federating autonomous ontologies (including TBox and ABox). The architecture contains two main components: The Federal Descriptor (FD) and the Federal Controller (FC) [28]. The first is responsible for identifying missing concept instantiation and identifying new alignments (based on previously defined ones). The latter is mainly responsible for executing SPARQL queries. More specifically, it comes with a Rule Selector module that is responsible for selecting only the subset of SWRL rules that allow returning results pertaining to the considered SPARQL query. In the following section we are presenting our conceptual approach to map between IFC to CityGML ontology (on a building scale).

\subsubsection{Conceptual Approach}

In this approach the ontologies remain independent from each other, no meta-model is created, defined or required. Only subparts of each $\mathrm{A}$ and $\mathrm{B}$ ontologies are aligned in other word, only some concepts and properties have been mapped as equivalent in the other ontology to create an interoperability schema (IS) 
between them, the below table gives an example of classes and attributes that are mapped between the two models (see Table .2 and Figure 4). As result CityGML model can easily and seamlessly fetch needed additional information from IFC model and visa-versa.

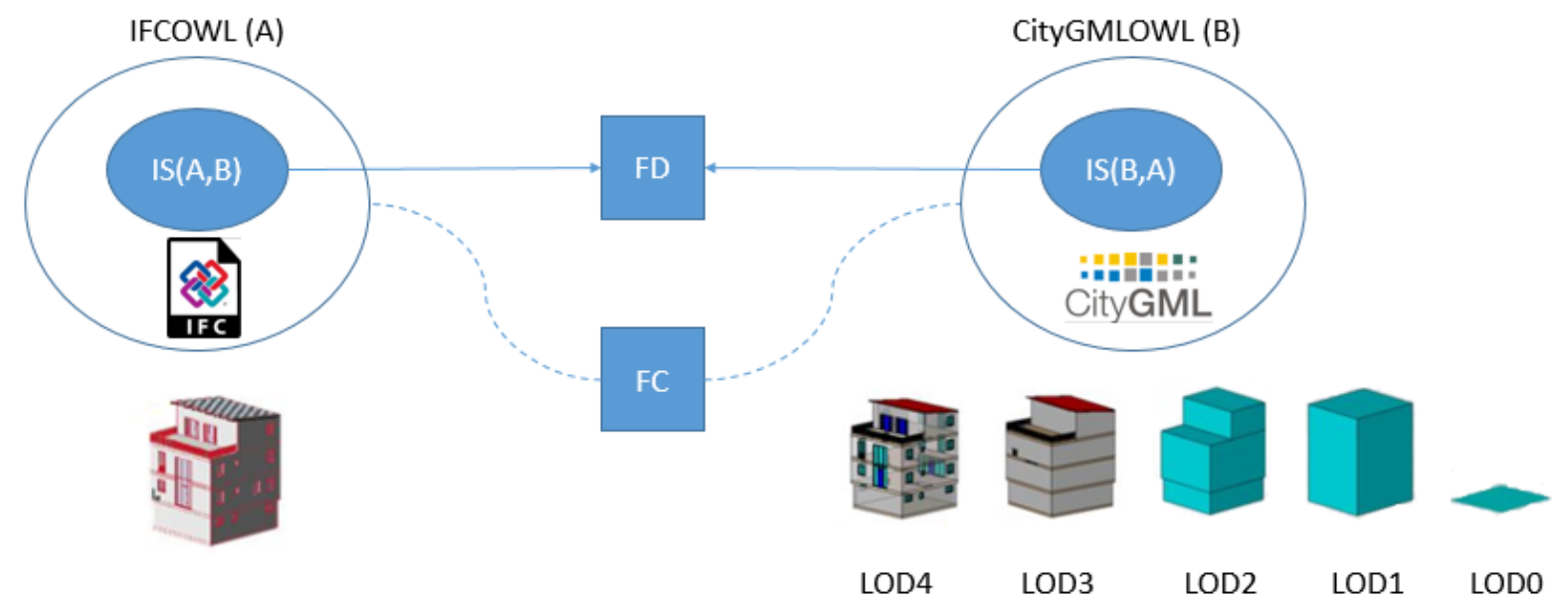

Fig. 4. Federation conceptual approach

Table. 2. Coupling between IFC and CityGML using first-order logic

\begin{tabular}{|c|c|c|}
\hline IFC OWL & Relationship & CityGML OWL \\
\hline IfcProject, IfcSite, IfcBuilding & Equivalent $(\approx)$ & _AbstractBuilding \\
\hline IfcSpace & $\approx$ & Room \\
\hline IfcWall & $\approx$ & WallSurface \\
\hline IfcSlab & $\approx$ & FloorSurface \\
\hline IfcRoof & $\approx$ & RoofSurface \\
\hline IfcRailing & $\approx$ & Railing \\
\hline IfcWindow & $\approx$ & Window \\
\hline IfcFurnishingElement & $\approx$ & BuildingFurniture \\
\hline IfcStair & $\approx$ & Stair \\
\hline IfcDoor & $\approx$ & Door \\
\hline IfcColumn & $\approx$ & column \\
\hline IfcBuildingAddress & $\approx$ & address \\
\hline IfcBuildingStorey & $\approx$ & BuildingPart \\
\hline IfcOpeningElement & $\approx$ & Opening \\
\hline IfcBeam & $\approx$ & Beam \\
\hline IfcBuildingStorey & $\approx$ & storeysAboveGround V \\
\hline Elevation & $\approx$ & StoreysBelowGround \\
\hline & & StoreyHeightsAboveGround \\
\hline storeHeightBelowGround \\
\hline IfcInternalOrExternalEnum $=$ True & $\approx$ & outerBuildingInstallation \\
\hline & & interBuildingInstallation \\
\hline
\end{tabular}

\subsection{Integration Approach}

Developing interoperability through an integrated approach means that there exists a common format for all models. Diverse models are built and interpreted using/against the common template. This format must be as detailed as the models themselves. In our context the integration approach will already detailed the building ontological model that describe all necessary concepts and properties needed 
to illustrate the building interior and exterior connection and will incorporate the necessary information from IFC and CityGML model (see Figure 5)

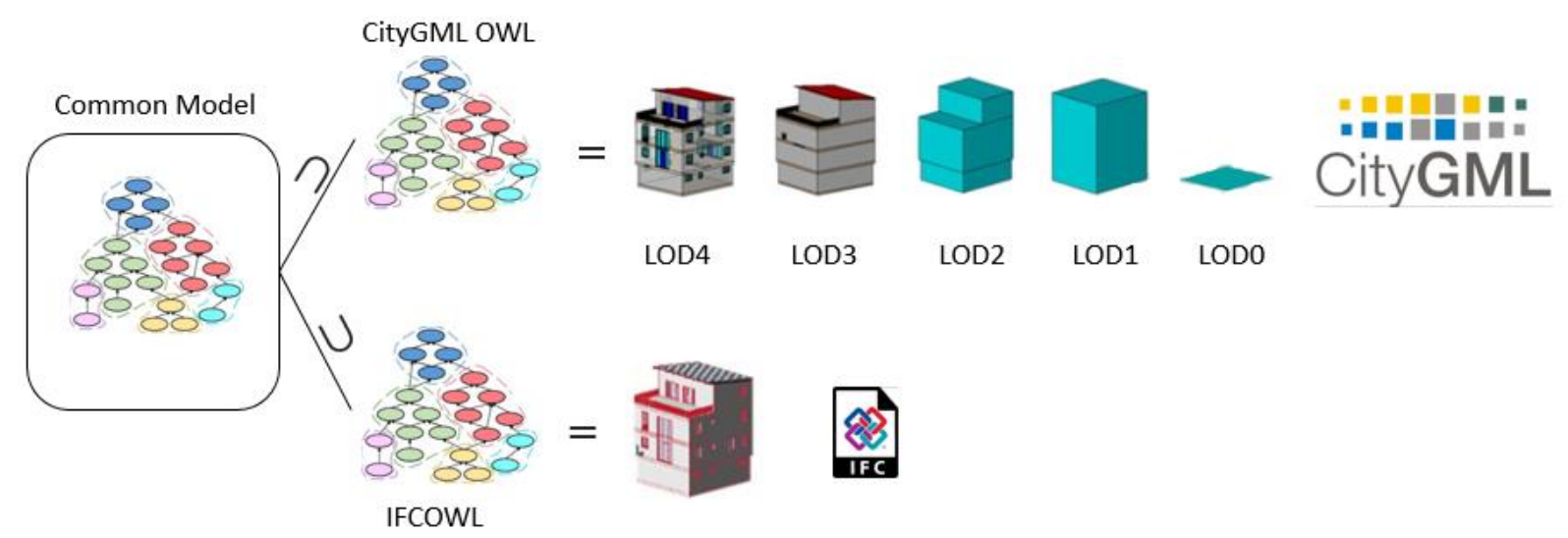

Fig. 6. Integration conceptual approach

\subsection{Unification Approach}

It means there is a common format but it only exists at the meta-model level. This format is not an executable entity as it is the case in integrated approach. Instead it provides a mean for semantic equivalence to allow mapping between models and applications. Using the
Meta-model, a translation between the constituent models is possible even though they might encounter loss of some semantics or information. In our context the unified approach will generate an extendable/ enhanced building CityGML model (Meta Model) where it contains the original CityGML building model and additional IFC information.

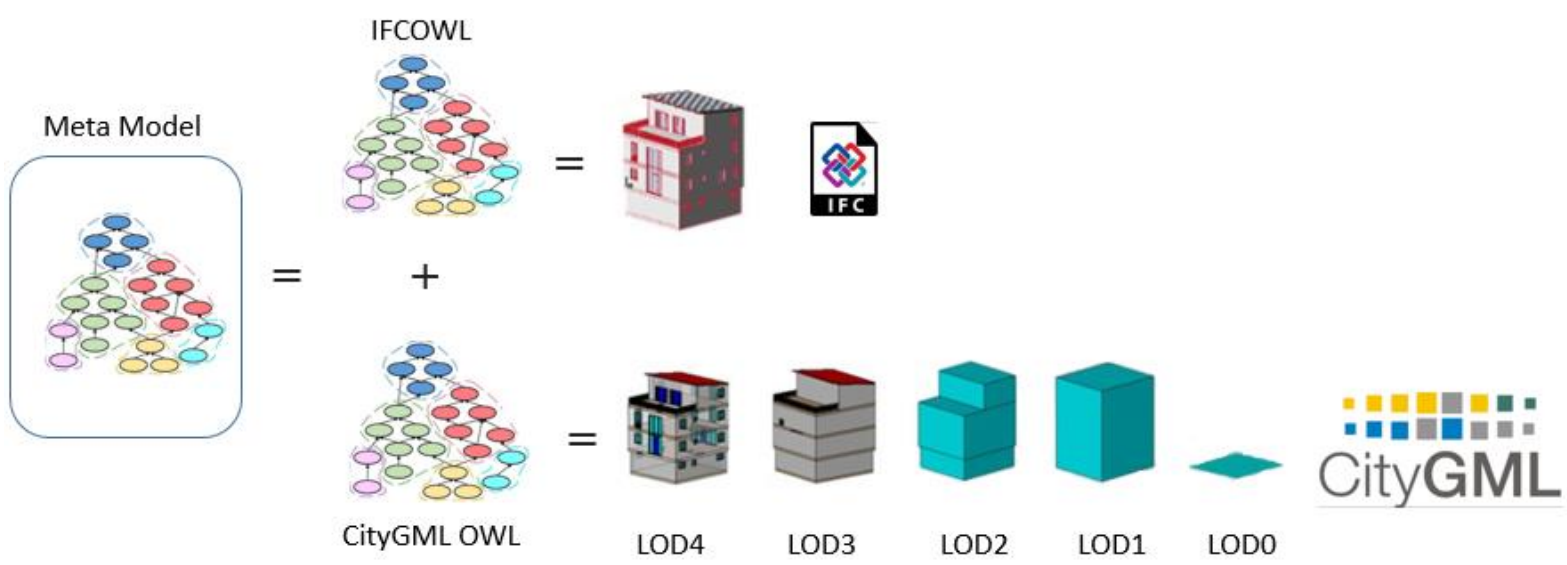

Fig. 7. Unified conceptual approach

\section{Semantic Checking in such Contexts}

Regardless of the approach chosen for reaching interoperability, a group of SPARQL queries that represent PLU (section 4.2) rule will be applied on the Knowledge base, extended model or the common model (section 5). As result we are going to obtain a compliance report that indicates the non-complaint elements of the urban model (see Figure 8). The only difference between the 3 models is that in the federation approach FC will control activate and deactivate the queries depending on the level of details defined, while the other approaches need to create an alignment between the generated model and PLU levels. 


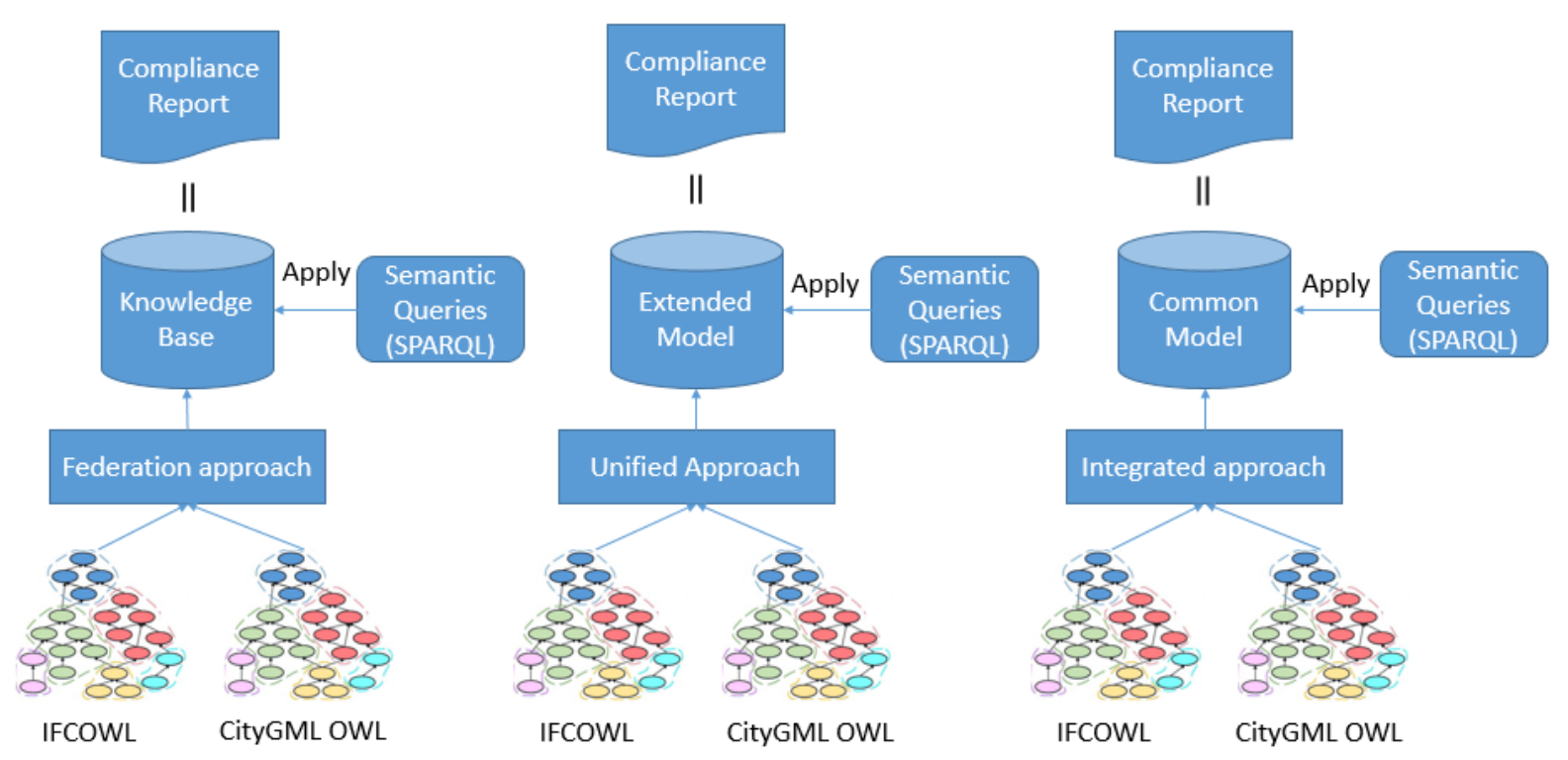

Fig. 8. Federation conceptual approach

\section{Conclusions and Future work}

In this article, we have introduced the semantic adaptation of PLU rules, illustrate all the possible approaches to achieve interoperability between BIM and GIS, and how a semantic checker is generated. The combination of the two methods helps us check the relationship and compliance of building elements with urban and construction rules. However our approach is semi-automatic and rely on human intervention. In future work we are going to evaluate the three approaches based on certain constraints such as: query response time, cost, etc. to find the most suitable one. In addition we are aiming to improve the approach presented in [29] which transform textual rules into semantic queries and finally connect PLU level with each multiscale model generated from the Interoperability approach.

\section{References}

[1] I. Ivan, and C. Ciurea, Quality characteristics of collaborative systems, in Proc. The Second International Conference on Advances in Computer-Human Interactions, vol. I, Cancun, Mexico, 2009, pp. 164168.

[2] P. Newman, \& A. Thornley, Urban Planning in Europe. 43

[3] Décret n ${ }^{\circ} 2015-1783$ du 28 décembre 2015 - art. Code de l'urbanisme - Articles R15118/20/. Code de l'urbanisme R151-18

[4] Décret n²015-1783 du 28 décembre 2015
- art.Code de l'urbanisme - Article R15120. Code de l'urbanisme R151-20

[5] Décret n ${ }^{\circ} 2015-1783$ du 28 décembre 2015 - art.Code de l'urbanisme - Article R15122. Code de l'urbanisme R151-22

[6] Décret n ${ }^{\circ} 2015-1783$ du 28 décembre 2015 - art.Code de l'urbanisme - Article R15124. Code de l'urbanisme R151-24

[7] ISO 16739:2013 Industry Foundation Classes (IFC) for data sharing in the construction and facilities management. (2013)

[8] ISO 10303: Industrial automation systems and integration - Product data representation and exchange - Part 11: Description methods: The EXPRESS language reference manual. (2004).

[9] G. Gröger, T.H. Kolbe, C. Nagel. \& K.H. Häfele, OGC City Geography Markup Language (CityGML) En- coding Standard. 344 (2012). 11

[10] ISO/IEC/IEEE 24765: Systems and software engineering - Vocabulary. (2017)

[11] H. Kubicek, R. Cimander, \& H.J. Scholl (2011). Layers of Interoperability. In H. Kubicek, R. Cimander, \& H. J. Scholl, Organizational Interoperability in E-Government (pp. 85-96).

[12] R. Hollenbeck, The IEEE 802.3 Standard (Ethernet): 24.

[13] J. Postel, Internet Protocol. Retrieved May 27, 2019, from https://tools.ietf.org/html/rfc791 
[14] J. Postel, Transmission Control Protocol. Retrieved May 27, 2019, from https://tools.ietf.org/html/rfc793

[15] Web Service Definition Language (WSDL). (2011). Retrieved May 13, 2019, from https://www.w3.org/TR/2001/NOTEwsdl-20010315

[16] SOAP Specifications. Retrieved August 1, 2019, from https://www.w3.org/TR/soap/

[17] ISO 11354: Advanced Automation technologies and their Application-requirements for establishing manufacturing enterprise process interoperability-Part 1: Framework for enterprise interoperability (2011).

[18] ISO 10303: Industrial automation systems and integration -- Product data representation and exchange -- Part 21: Implementation methods: Clear text encoding of the exchange structure. (2016)

[19] R. Studer, R. Benjamins, and D. Fensel. Knowledge engineering: Principles and methods. Data and Knowledge Engineering, 25 (1-2):161-198, 1998.

[20] Y. Deng, J.C.P. Cheng, \& C. Anumba, (2016). Mapping between BIM and 3D GIS in different levels of detail using schema mediation and instance comparison. Automation in Construction, 67, 121.

[21] C. Mignard \& C. Nicolle, Merging BIM and GIS using ontologies application to urban facility management in ACTIVe3D. Computers in Industry, 65(9), 1276-1290. https://doi.org/10.1016/j.compind.2014.07.008

[22] G. Falquet, N.Ghoula \& C. Métral, Towards semantically enriched 3D city models an ontology-based approach. 6 (2009)

[23] G. Floros, I. Pispidikis, \& E. Dimopoulou, (2017). INVESTIGATING INTEGRATION CAPABILITIES BETWEEN IFC AND CITYGML LOD3 FOR 3D CITY MODELLING. ISPRS - International Archives of the Photogrammetry,
Remote Sensing and Spatial Information Sciences, XLII-4/W7, 1-6. https://doi.org/10.5194/isprs-archivesXLII-4-W7-1-2017

[24] Resource Description Framework (RDF): Concepts and Abstract Syntax. Available at: https://www.w3.org/TR/rdf-concepts/. (Accessed: 21st February 2019)

[25] T. Gruber. A translation approach to portable ontology specifications. Technical Report No. KSL 92-71, Knowledge Systems Laboratory, Stanford University, Stanford, CA.

[26] F. Fonseca, G. Câmara, \& A. Monteiro. A framework for measuring the interoperability of geo-ontologies. Spatial Cognition and Computation, 6(4), 307-329.

[27] Mena E., Kashyap V., Sheth A. \& Illarramendi A. OBSERVER: An approach for query processing in global information systems based on interoperation across pre-existing ontologies. Paper presented at the First IFCIS International Conference on Cooperative Information Systems (CoopIS'96), Brussels, Belgium.

[28] T.M. Farias, A. Roxin. \& C. Nicolle. FOWLA, A Federated Architecture for Ontologies. in Rule Technologies: Foundations, Tools, and Applications (eds. Bassiliades, N., Gottlob, G., Sadri, F., Paschke, A. \& Roman, D.) 9202, 97-111 (Springer International Publishing, 2015).

[29] K.R. Bouzidi, B. Fies, C. Faron-Zucker, A. Zarli, \& N.L. Thanh, Semantic Web Approach to Ease Regulation Compliance Checking in Construction Industry. Future Internet 4, 830-851 (2012).

[30] J. Zhang, \& N.M. El-Gohary, Automated Information Transformation for Automated Regulatory Compliance Checking in Construction. Journal of Computing in Civil Engineering 29, B4015001 (2015).

[31] R. Carnap. Meaning and Necessity - A Study in Semantics and Modal Logic. The University of Chicago Press, second edition, 1956 


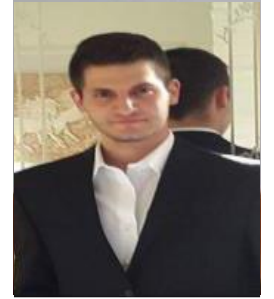

Elio HBEICH Has graduated from University of Burgundy in 2018 with a master degree in database and artificial intelligence. Currently, a first-year Ph.D. student working on achieving interoperability between BIM and GIS models through semantic technologies and applying semantic queries (SPARQL) to creating a semantic checker that verify the compliances of building models, cities and territories.

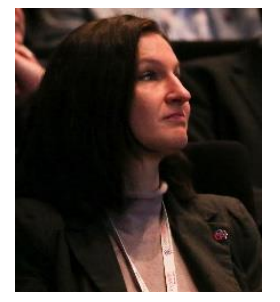

Ana ROXIN holds a Computer Science engineering diploma along with a $\mathrm{PhD}$ in Computer Science, obtained in 2009 from UTBM (). Dr. Ana ROXIN is an associate professor at the department of Computer Science of the University of Burgundy, and full member of the LIB laboratory (EA7534). She was involved in several national and European projects (e.g. EU FP7 ASSET, TELEFOT) addressing pushing sensitive information to the user. Her main research interests concern: knowledge engineering for BIM, semantic interoperability and reasoning in a Big Data context. She is a member of the French national standardization organization, AFNOR, more specifically of the PPBIM commission dealing with standards for BIM products and processes. She represents France in mirror committees in international standardization organizations e.g. ISO and CEN. She is also a member of building SMART International, the Open Geospatial Consortium (OGC) and the W3C.

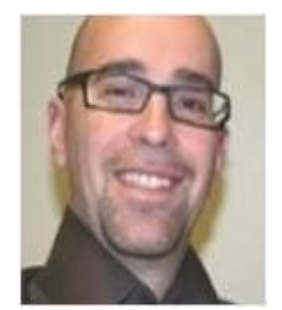

Nicolas BUS has graduated the Engineer High School (Ecole Nationale Supérieure de Arts et Métiers) in 1997. He hold a general engineer diploma. He has firstly worked as a computer scientist specialized in numerical computation and databases in various domain such as aerospace, construction industry and finance. He joined CSTB (Centre Scientific et Technique du Bâtiment) in 2001 as a research engineer developing information systems for the French ministry of housing and environnement. For the past few years he has been involved in various European projects on modeling construction and architecture systems and processes. Nicolas BUS is currently leading the Emerging Technologies Research Program at CSTB. 